\title{
The Effect of Yoga on the Psychological Distress of Cancer Patients: An Analytical Study
}

\author{
Dr. Roshan Anie Alex
}

UGC Post Doctoral Research Fellow

\begin{abstract}
Since holistic approaches to healing are rising, yoga therapy for cancer patients and cancer survivors is emerging as one of the more successful methods for combating the physical discomfort of cancer and cancer treatment. Aim: The study is taken up to probe whether practising yoga effect the level of depression, anxiety, stress and psychological distress of the cancer patients. Design: An experimental group design was used. Experimental group (yoga group (n=15)) and control group (n=15) were assigned for the study. The dependent variables were the participants' pre-post difference in scores of depression, anxiety, stress and psychological distress. The independent variables were yoga. The study investigated the effect of yoga on the psychological distress of cancer patients. Participants: The participants of the present study consisted of 30 cancer patients selected from hospitals in Trivandrum using purposive sampling method. 15 cancer patients were selected for yoga based on their willingness for attending the intervention. 15 cancer patients were kept as control group. Measures: The tools used were Depression Anxiety Stress Scale (DASS 21) developed by Lovibond, S.H. \& Lovibond, P.F (1995); and Yoga instructions by an expert yoga instructor. The yoga practice included Surya Namaskar, Vakrasana, Trigonasana, Marjarasana, Uttanpadasana, Sankatasana, Pavanmuktasana, Tadasana and Pranayama. Data analysis: Wilcoxon Paired Signed Rank Test was done to interpret the data. Findings: The findings of the present study proved that yoga have significant effect in reducing the psychological distress of cancer patients. It is hoped that the outcome of this study will be beneficial for cancer patients.
\end{abstract}

Keywords: Yoga, cancer, psychological distress

\section{Introduction}

When battling cancer, the worst part is not just the symptoms of the disease itself, but often the discomfort and debilitating fatigue brought on from cancer treatments. Whether faced with the scar-tissue of surgery or ongoing nausea and weakness from chemotherapy or radiation, cancer patients endure a long road of physical trials. But as many cancer patients and cancer survivors are discovering, there are ways to strengthen their bodies and deal with the uncomfortable side-effects of treatment, both during and after treatment.

As more holistic approaches to healing is growing, yoga therapy for cancer patients and cancer survivors is emerging as one of the more successful methods for combating the physical discomfort of cancer and cancer treatment. Gentle yoga poses for cancer patients can work magic on many levels. First of all, yoga used as therapy for cancer can help clear out toxins accrued during cancer treatment more effectively. Yoga asanas stimulate not just muscles, but also increases blood flow, balances the glands and enhances the lymphatic flow in the body, all of which enhances the body's internal purification processes. The deep, relaxing breathing often emphasized in yoga for cancer therapy also increases the current of oxygen-rich blood to the cells, delivering vital nutrients to tired cells and further clearing out toxins.

For those enduring chemotherapy and radiation, yoga for cancer provides a means to strengthen the body, boost them immune system, and produce a much-sought-after feeling of well-being. For those recovering from surgery, such as that for breast cancer, yoga can help restore motion and flexibility in a gentle, balanced manner. Yoga for cancer survivors and patients also provides an internal anchor of calm. Many practicing yoga therapy have discovered an interesting, subtle benefit, an increased awareness of a great, internal stillness and sense of unity. They've found, at the most fundamental level of their own consciousness, a sense of true health and vitality that spills over into other aspects of life (YogaUonline, 2015).

Yoga, along with meditation, can alleviate depression; anxiety and insomnia to help patients become "emotionally fit." Medical research is revealing what cancer centers and yogic practitioners have long known, namely that "patients at all stages of health, including cancer survivors, can benefit from yoga. And the benefits are both physical and emotional." Some of the benefits from yoga are as follows:

1. Yoga Helps Manage Depression, Fear and Anxiety: Depression and an acute fear of death can be prevalent in patients suffering through the emotional strain of a cancer diagnosis. Undergoing invasive or rigorous treatments may also heighten anxiety. "Yogic breathing, defined as a manipulation of breath movement, has been shown to positively affect immune function, autonomic nervous system imbalances, and psychological or stressrelated disorders." In other words, guided breathing exercises enrich the respiratory system to regulate nerves that can deeply calm both mind and body. Yoga activates this relaxation response and can thus help relieve feelings of anxiety. With the aid of supported inversions to increase circulation and guided meditation/deep breathing to let go of grief, fear, and foreboding, you actually re-pattern and calm stress cycles.

2. The "Mood Boost" Effect: Exercise produces endorphins and endorphins improve mood. A regular yoga practice, no matter how gentle the movements, allows the body to release endorphins so can instantly experience a positive boost in mood. Several studies also suggest that yoga can increase the level of gamma-aminobutyric acid, a neurotransmitter that plays a key role in regulating the nervous system and managing mood and outlook. 


\section{International Journal of Science and Research (IJSR) \\ ISSN (Online): 2319-7064 \\ Index Copernicus Value (2013): 6.14 | Impact Factor (2014): 5.611}

3. Help to Manage Physical Pain: Beside the well known and painful physiological side effects of cancer treatment, emotional stress can also produce physical pain. Moderate, appropriately modified physical activity aids in managing the physical pain that can be experienced during treatment, and research has shown that women who practice yoga specifically when in recovery report reduced pain and stress. Gentle guided movements, can help to expand range of motion, improve circulation, and relax muscle tension to speed up the recovery process.

4. Yoga Community Can Provide Support: A support system is crucial to coping with the emotional toll of cancer, not only for those suffering from the disease but for their loved ones as well (YogaUonline, 2015)

Studies show that yoga could help to reduce anxiety, depression, fatigue and stress for some patients. And it improved the quality of sleep, mood and spiritual well being for some people. Overall yoga may be associated with some positive effects on psychological well being for people with cancer. In patients with breast cancer it is found that yoga helped to reduce distress, anxiety, depression and tiredness (fatigue). It also helped to improve quality of life, emotional wellbeing and social wellbeing. Yoga reduced tiredness (fatigue) in women with breast cancer. Some studies seem to show that yoga may be able to reduce hot flushes in women with breast cancer. 1 small trial showed that people with lymphoma had fewer sleep disturbances, fell asleep more quickly, and slept for longer after a 7 week yoga programme. But we need bigger studies to confirm all these findings. (A small individual study in the USA in 2012 found that) Studies have shown that a structured yoga practice during cancer treatment can radically improve physical symptoms such as pain and fatigue. Yoga reduces stress and anxiety which in turn reduces heart rate, lowers blood pressure, and eases respiration. What's more, patients who practiced yoga were also less sensitive to pain than subjects who did not, and therefore better able to tolerate treatment (Harvard Medical School Mental Health Letter, 2009).

Having noticed the benefits of yoga as an alternative therapy, this study is taken up to probe whether practising yoga effect the level of depression, anxiety, stress and psychological distress of the cancer patients. It is hoped that the outcome of this study will be beneficial for cancer patients. Effective distress management techniques can then be taken up to improve the well-being of those who suffering from cancer diseases.

\section{Method}

a) Design: An experimental group design was used. Experimental group (yoga group $(n=15)$ ) and control group $(n=15)$ were assigned for the study. The dependent variables were the participants' pre-post difference in scores of depression, anxiety, stress and psychological distress. The independent variables were yoga. The study investigated the effect of yoga on the psychological distress of cancer patients.

b) Participants: The participants of the present study consisted of 30 cancer patients selected from hospitals in
Trivandrum using purposive sampling method. 15 cancer patients were selected for yoga based on their willingness for attending the intervention. 15 cancer patients were kept as control group.

c) Measures: The tools used were Depression Anxiety Stress Scale (DASS 21) developed by Lovibond, S.H. \& Lovibond, P.F.(1995); and Yoga instructions by an expert yoga instructor. The yoga practice included Surya Namaskar, Vakrasana, Trigonasana, Marjarasana, Uttanpadasana, Sankatasana, Pavanmuktasana, Tadasana and Pranayama

d) Data analysis: Wilcoxon Paired Signed Rank Test was done to interpret the data.

\section{Results and Discussions}

a. Comparison between pre test and post test scores of psychological distress in control and experimental groups.

The data on psychological distress scores were collected before and after the intervention and calculated the mean and SD. The significant pre and post test difference of psychological distress scores of experimental and control groups were tested using Wilcoxson Signed Rank Test. The details are given in Table 1

Table 1: Comparison between pre and post test scores in psychological distress in control and experimental groups

\begin{tabular}{|c|c|c|c|c|c|}
\hline Groups & Variables & Mean & $S D$ & $Z$ & $P$ \\
\hline \multirow{5}{*}{ Yoga Group } & D1 & 12.8000 & 6.9857 & 4.043 & $.002^{* *}$ \\
\cline { 2 - 6 } & D2 & 9.0667 & 4.9391 & & \\
\cline { 2 - 6 } & A1 & 8.6667 & 4.2887 & 2.138 & .255 \\
\cline { 2 - 6 } & A2 & 7.8667 & 2.7265 & & \\
\cline { 2 - 6 } & S1 & 11.7333 & 4.7115 & 3.467 & $.001^{* *}$ \\
\cline { 2 - 6 } & S2 & 8.1333 & 2.7265 & & \\
\cline { 2 - 6 } & Pd1 & 29.2000 & 10.347 & 3.342 & $.001^{* *}$ \\
\cline { 2 - 6 } & Pd2 & 21.0667 & 5.1312 & & \\
\hline \multirow{5}{*}{ Control Group } & D1 & 8.6000 & 3.3975 & .531 & .596 \\
\cline { 2 - 6 } & D2 & 8.8000 & 2.7568 & & \\
\cline { 2 - 6 } & A1 & 7.7333 & 2.2824 & .630 & .528 \\
\cline { 2 - 6 } & A2 & 8.3333 & 3.0861 & & \\
\cline { 2 - 6 } & S1 & 10.2667 & 3.3267 & .974 & .330 \\
\cline { 2 - 6 } & S2 & 10.9333 & 2.6851 & & \\
\cline { 2 - 6 } & Tp1 & 26.6000 & 5.9618 & .797 & .425 \\
\cline { 2 - 6 } & Tp2 & 28.0667 & 6.5843 & & \\
\hline
\end{tabular}

Note: $* *$ p value is significant at 0.01 level

$*$ p value is significant at 0.05 level

Table 1, shows that the pre-post test difference in depression $(\mathrm{p}=.002)$, stress $(\mathrm{p}=.001)$ and psychological distress $(\mathrm{p}=.001)$ was significant in yoga group, while the pre-post test difference in anxiety $(\mathrm{p}=.255)$ was not significant in yoga group. The pre-post test difference in depression $(\mathrm{p}=.596)$, anxiety $(\mathrm{p}=.528)$, stress $(\mathrm{p}=.330)$ and psychological distress $(\mathrm{p}=.425)$ was not significant in control group.

The further interpretations of the results with regard pre and post scores of depression, anxiety, stress and psychological distress of experimental groups and control groups are as follows:

\section{Yoga Group}

The mean values showed that cancer patients those who undergone yoga had significantly lower level of depression, 


\section{International Journal of Science and Research (IJSR) \\ ISSN (Online): 2319-7064}

Index Copernicus Value (2013): 6.14 | Impact Factor (2014): 5.611

stress and psychological distress than those who do not undergone the yoga. Even though, pre-post test difference in anxiety was not significant, a closer look on the mean values showed that cancer patients those who undergone yoga had significantly lower level of anxiety than those who do not undergone the yoga. The results clearly indicate that yoga have a significant role in reducing the psychological distress of cancer patients.

\section{Control Group}

Though the pre-post difference in the scores of depression, anxiety, stress and psychological distress was not seen to be significant, a further examination of the mean scores of the psychological distress variables indicate that the post test scores of depression, anxiety, stress and psychological distress is higher than those of pre test.

The findings of the study evidently emphasis the need for the implication of an intervention program in cancer patients for reducing their psychological distress.

\section{Conclusion}

The findings of the present study proved that yoga have significant effect in reducing the psychological distress of cancer patients. When the implication of the present study is considered, it can be said that all the findings of the present investigation can be of much help for cancer patients and others directly or indirectly linked to the wellbeing of cancer patients. It will be beneficial for cancer patients if they use yoga as an alternative therapy along with their medicine. These findings can be immense worth for oncologists, psychologists, and yoga therapists. It is suggested that major hospitals should make earnest endeavour to set up special departments consisting of oncologists, psychologists, yoga therapists. A general awareness about the therapeutic effects of yoga has to be created by means of mass communication, in order to be more beneficial to common people. The investigator would be grateful, if the findings of the present investigation are used for advance research and favourable for those implicated in the wellbeing of cancer patients.

\section{References}

[1] Gregson, O., \& Looker,T. (1994). “ The biological basis of stress management". British Journal of Guidance and counseling, 22 (1). 1-26.

[2] Hirsch S, Meckes D. (2000). Treatment of the whole person: incorporating emergent perspectives in collaborative medicine, empowerment, and music therapy. J PsychosocOncol; 18(2):65-77

[3] Lovibond, S.H.;Lovibond, P.F. (1995). Manual for the Depression Anxiety Stress Scales (2nd ed.). Sydney: Psychology Foundation.

[4] Schneiderman, Neil (2003). Perceived stress management skill mediates the relationship between optimism and positive mood following radical prostatectomy. Health Psychology, 22(2), 220-222

[5] The Harvard Heart Letter (2009). Harvard health publications, Harvard Medical School.

[6] YogaUonline.com, (2015). How Yoga Helps Cancer Patients and Cancer Survivors. In https://www.yogauonline.com/yogau-wellness-blog/howyoga-helps-cancer-patients-and-cancer-survivors 